\title{
PARTICIPACIÓN FEMENINA EN LOS CONSEJOS DE ADMINISTRACIÓN DE LAS EMPRESAS: UNA APROXIMACIÓN A LA PROPUESTA DE DIRECTIVA DE 14 DE NOVIEMBRE DE 2012 RELATIVA AL EQUILIBRIO ENTRE HOMBRES Y MUJERES EN LAS EMPRESAS
}

\author{
Maoly Carrasquero Cepeda
}

\begin{abstract}
SUMARIO: 1. INTRODUCCIÓN. 2. EQUIDAD DE GÉNERO EN LOS CONSEJOS DE ADMINISTRACIÓN DE LAS SOCIEDADES MERCANTILES ESPAÑOLAS. 2.1. Antecedentes. 2.2. Ley de Orgánica de Igualdad 3/2007. 3. REgULAción normativa europea. 3.1. Antecedentes. 3.2. Propuesta de Directiva. 4. COMPARATIVA DE AMBOS TEXTOS NORMATIVOS. 5. CONClUSIONES. 6. BIBLIOGRAFÍA.
\end{abstract}

\section{INTRODUCCIÓN}

La representación femenina en los consejos de administración de las empresas es un tema de gran actualidad dadas las diferentes iniciativas legislativas y regulatorias que se han producido últimamente en algunos Estados miembros de la Unión Europea (UE).

Hasta la fecha, Noruega, Francia, Italia y Bélgica han introducido un sistema de cuotas jurídicamente vinculante, que incluye sanciones. Por su parte, Países Bajos y España también han adoptado medidas legislativas que prevén cuotas en favor del género menos representado, aunque no se sanciona su incumplimiento. En Dinamarca, Finlandia, Portugal, Grecia, Austria y Eslovenia sólo se aplican cuotas para la representación femenina en los consejos de administración de las empresas de titularidad pública. En los demás Estados, que no han consagrado ninguna medida obligatoria, se han adoptado acciones de carácter voluntario que promueven la representación de las mujeres en los puestos superiores de gestión (por ejemplo: Dinamarca, Alemania, Luxemburgo, Polonia, Suecia y el Reino Unido). 
Sin embargo, pese a los avances realizados, el logro de la igualdad efectiva de hombres y mujeres en el sector económico todavía está muy lejos de alcanzarse. En toda la UE, las mujeres apenas consiguen el 5\% de participación en los puestos más elevados ${ }^{1}$.

En el caso concreto de España, la situación es realmente preocupante. A un año de finalizar el plazo otorgado por la Ley para alcanzar una cuota del $40 \%$, apenas se llega al $15 \%$ de participación femenina en los consejos de administración de las mayores sociedades mercantiles ${ }^{2}$.

A partir de este contexto, el objeto del presente trabajo consiste en analizar las diferentes iniciativas legislativas que se han elaborado por parte de la UE y de España para mejorar la situación de la mujer en la toma de decisiones económicas. Así, en una primera parte, se realizará un breve estudio del artículo 75 de la Ley Orgánica 3/2007, de 22 de marzo, para la igualdad efectiva de mujeres y hombres, que consagra el principio de presencia equilibrada en los consejos de administración de las sociedades mercantiles españolas. En segundo lugar, se examinará la reciente Propuesta de Directiva destinada a mejorar el equilibrio de género entre los administradores no ejecutivos de las empresas cotizadas, en la que se establece una cuota obligatoria del $40 \%$ de mujeres en los consejos de administración de las sociedades cotizadas. Y, por último, se indicarán las diferencias y similitudes más notables entre ambas disposiciones normativas.

\section{EQUIDAD DE GÉNERO EN LOS CONSEJOS DE ADMINISTRACIÓN DE LAS SOCIEDADES MERCANTILES ESPAÑOLAS}

\subsection{Antecedentes}

En el caso específico español, se debe citar, como principal antecedente de la cuestión la referencia a la diversidad de género en los consejos de administración que realiza el Código Unificado de Buen Gobierno de las sociedades cotizadas (CUBG), aprobado por el Consejo de la Comisión Nacional del Mercado de Valores, con fecha de 22 de mayo de $2006^{3}$.

\footnotetext{
${ }^{1}$ Este porcentaje hace referencia únicamente a las mujeres que ocupan el cargo de presidentas de consejos de administración en las mayores empresas de la Unión. Vid.: http://ec.europa.eu/justice/gender-equality/gender-decision-making/database/business-finance/supervisory-board-board-directors/index_en.htm (visto el 02.06.2014).

${ }^{2}$ Vid.: http://ec.europa.eu/justice/gender-equality/gender-decision-making/database/ business-finance/supervisory-board-board-directors/index_en.htm (visto el 02.06.2014).

${ }^{3}$ El CUBG es elaborado con el objetivo de armonizar y actualizar las recomendaciones de los informes Olivencia (1998) y Aldama (2003) sobre buen gobierno de las sociedades cotizadas, además de formular recomendaciones adicionales que se consideren necesarias, como es el caso de la equidad de género en los Consejos de administración. Vid.: Campuzano Laguillo, A., «La presencia equilibrada de hombres y mujeres en los Con-
} 
El CUBG contempla una serie de reglas de fomento (recomendaciones) que no suponen una vinculación directa para las sociedades afectadas, puesto que se enmarca dentro de la denominada responsabilidad social empresarial ${ }^{4}$.

Precisamente, se establece en su Recomendación 15:

«Que cuando sea escaso o nulo el número de consejeras, el Consejo explique los motivos y las iniciativas adoptadas para corregir tal situación; y que, en particular, la Comisión de nombramientos vele para que al proveerse nuevas vacantes:

a) Los procedimientos de selección no adolezcan de sesgos implícitos que obstaculicen la selección de consejeras;

b) La compañía busque deliberadamente, e incluya entre los potenciales candidatos, mujeres que reúnan el perfil profesional buscado» ${ }^{5}$.

Plantea esta recomendación una formulación del modelo comply or explain (cumplir o explicar) típica de las reglas de Buen Gobierno: se invita, por una parte, a corregir la escasez de consejeras mediante la eliminación de «sesgos implícitos» en los procedimientos de selección y la «búsqueda deliberada» de mujeres candidatas y, por otra, en la medida en que se mantenga la escasez, se exigen explicaciones sobre las acciones que se han adoptado para su superación ${ }^{6}$.

sejos de administración de las sociedades mercantiles», en GARCÍA-PERROTE ESCARÍN, I. Y MERCADER Uguina, J. (coords.): La Ley de igualdad: consecuencias prácticas en las relaciones laborales y en la empresa, Lex Nova, Valladolid, 2007 pp. 190; MATEU de Ros Cerezo, R., El Código Unificado de Gobierno Corporativo, Thomson Aranzadi, Navarra, 2007, p. 13.

${ }^{4}$ La responsabilidad social se ha definido como «La integración voluntaria, por parte de las empresas, de las preocupaciones sociales y medioambientales en sus operaciones comerciales y sus relaciones con sus interlocutores». Libro Verde de la Comisión de las Comunidades Europeas, «Fomentar un marco europeo para la responsabilidad social de las empresas», Bruselas 18.7.2001 COM (2001) 366 final. Posteriormente, la Comunicación de la Comisión añade a esta definición que «La responsabilidad social consiste también en gestionar los cambios dentro de la empresa de una manera socialmente responsable, lo que ocurre cuando una empresa procura reconciliar los intereses y las necesidades de distintas partes de manera aceptable para todas ellas». Comunicación de la Comisión relativa a la responsabilidad social de las empresas: una contribución al desarrollo sostenible, Bruselas, 2.7.2002 COM (2002) 347 final.

${ }^{5}$ En el actual Código Unificado de Buen Gobierno de las sociedades cotizadas (junio 2013) esta Recomendación es recogida en términos similares, salvo por el curioso hecho de que se suprime la obligación impuesta al Consejo para que, cuando sea escaso o nulo el número de consejeras, explique las iniciativas adoptadas a fin de corregir tal situación.

${ }^{6}$ En este sentido, véase: Senent Vidal, M. en García Ninet, J. (dir.) Comentarios a la Ley de Igualdad, CISS, Bilbao, 2007, pp. 543-544; Rodríguez González, A.: Mujeres en los Consejos de administración de las sociedades mercantiles: una aproximación al Derecho francés, Valladolid, 2011, p. 10. 
El objetivo de esta disposición no es otro que los procesos de selección se articulen de forma que no exista suposición ninguna de discriminación por género, procurando que sea la cualificación y la competencia la que determine el ingreso del consejero, sin distinguir entre hombres y mujeres.

Aunque la mayoría de las empresas y profesionales se mostraron partidarias de la inclusión de esta disposición, algunos expresaron su disconformidad argumentando, entre otras cosas, que la igualdad de género es una política pública ajena de los intereses privados de las compañías cotizadas (Instituto de Consejeros Administradores). Por este motivo, los criterios de eficiencia en los que sustenta la medida, pueden lograrse a través de requisitos de capacitación y habilitación, que no tienen nada que ver con cuestiones de sexo (Asociación Española de Accionistas Minoritarios de Empresas Cotizadas) $)^{7}$.

Estas alegaciones fueron superadas y logró mantenerse la recomendación sobre la diversidad de género en los consejos de administración que, junto con la posterior aprobación de la Ley Orgánica 3/2007, de 22 de marzo, para la igualdad efectiva de mujeres y hombres (LOI), supuso un gran avance en las políticas de igualdad españolas.

\subsection{Ley Orgánica 3/2007 para la igualdad efectiva de mujeres y hombres}

La LOI mantiene la concepción de las cuotas de género en las empresas como una medida concreta de la responsabilidad social corporativa y buen gobierno. Así, en el Título VII (relativo a la igualdad en la responsabilidad social de las empresas) se incorpora el artículo 75, titulado «Participación de las mujeres en los consejos de administración de las sociedades mercantiles», el cual indica:

«Las sociedades obligadas a presentar cuenta de pérdidas y ganancias no abreviada procurarán incluir en su Consejo de administración un número de mujeres que permita alcanzar una presencia equilibrada de mujeres y hombres en un plazo de ocho años a partir de la entrada en vigor de esta Ley.

Lo previsto en el párrafo anterior se tendrá en cuenta para los nombramientos que se realicen a medida que venza el mandato de los consejeros designados antes de la entrada en vigor de esta Ley».

Es evidente que el legislador aspira con esta medida (de carácter no imperativo $^{8}$ ) a que las propias empresas contribuyan a aumentar el escaso

7 Para un análisis más detallado de todas las objeciones que se expresaron véase: Márquez Lobillo, P., «Consideraciones acerca de la incorporación de la mujer a los consejos de administración tras la Ley Orgánica de Igualdad», en Revista de Derecho de Sociedades, núm. 32, 2009, p. 281.

${ }^{8}$ No existe un consenso en la doctrina sobre la naturaleza de esta medida, sin bien es cierto que la mayoría de los autores la consideran como una mera recomendación, 
número de representación femenina en sus consejos de administración, a fin de lograr la presencia equilibrada entre hombres y mujeres. Este equilibrio se define como que las personas de cada sexo no superen el $60 \%$ ni sean menos del $40 \%{ }^{9}$.

A tal efecto, se prevé un plazo de ocho años a partir del año 2007 (cuando entró en vigor la norma $)^{10}$, sin perjuicio de que se tenga en cuenta para los nombramientos que se realicen a medida que se venza el mandato de los consejeros designados ${ }^{11}$. Este período de tiempo, sin embargo, no rige para el caso de los nombramientos en los consejos de administración que puedan efectuar los organismos públicos, ya que, para ellos el mandato es imperativo y han de cumplir con la medida desde el mismo momento de entrada en vigor de la Ley ${ }^{12}$.

existe una opinión minoritaria que la enmarca dentro de las denominadas obligaciones de medios. Así, EMBRID IRUJo, J., «Los aspectos mercantiles de la Ley Orgánica para la igualdad efectiva de mujeres y hombres», en AA.VV. Comentarios a la Ley Orgánica 3/2007, de 22 de marzo para la igualdad efectiva de mujeres y hombres, La Ley, Madrid, 2008, p. 340, señala que «nos encontramos ante una obligación de medios, cuyo satisfactorio cumplimiento está vinculado al empleo de la diligencia por parte de las sociedades afectadas por la norma; así, estas sociedades harán, dentro del marco de su diligencia, todo lo posible para que se consiga, dentro del plazo establecido en la Ley, la ya referida presencia equilibrada de mujeres y hombres en el Consejo de administración, sin que la ausencia de este resultado, per se, haya de considerarse incumplimiento de su obligación».

${ }^{9}$ La disposición adicional primera de la LOI define el principio de presencia o composición equilibrada como: «La presencia de mujeres y hombres de forma que, en el conjunto a que se refiera, las personas de cada sexo no superen el sesenta por ciento ni sean menos del cuarenta por ciento».

${ }^{10}$ El Anteproyecto de LOI inicialmente contenía una propuesta un poco más exigente, al señalar que las empresas contaban con un plazo de cuatro años, en los que debían incorporar, al menos, un diez por ciento anual de mujeres. Empero, dicha redacción fue modificada y se aumentó el plazo a ocho años. Víd.: SENENT VIDAL, M. en GARCía Ninet, J. (dir.) Comentarios a la Ley de Igualdad... op. cit., p. 551 ss.

${ }^{11}$ No parece lógico el planteamiento que hace aquí el legislador, toda vez que parece haber descartado ciertos supuestos de que se produzca una vacante en el Consejo por cuestiones distintas del vencimiento del mandato, tales como: dimisión del consejero, revocación de su cargo por la Junta General, fallecimiento, entre otras. Sobre este punto véase: EMBRID IrUJo, J., «Los aspectos mercantiles de la Ley Orgánica para la igualdad efectiva de mujeres y hombres»... op. cit., p. 344; SENENT VIDAL, M., «El principio de presencia equilibrada en el ámbito del Derecho privado», en El levantamiento del velo: las mujeres en el Derecho privado. García Rubio, M. y Valpuesta Fernández, M. (dirs.), Valencia, 2011, p. 1302.

${ }_{12}$ Así lo establece el artículo 54.2 de la LOI: «La Administración General del Estado y los organismos públicos vinculados o dependientes de ella observarán el principio de presencia equilibrada en los nombramientos que le corresponda efectuar en los consejos de administración de las empresas en cuyo capital participe». 
Por otra parte, en cuanto al ámbito subjetivo de aplicación del artículo 75 LOI, el mismo se limita a las sociedades mercantiles obligadas a presentar cuenta de pérdidas y ganancias no abreviada ${ }^{13}$, excluyendo así a las pequeñas y medianas empresas ${ }^{14}$.

También se exceptúa del alcance de la disposición reseñada, aquellas sociedades que tengan organizada su estructura administrativa mediante cualquiera de las otras formas previstas en el ordenamiento jurídico, que no sea específicamente el consejo de administración, a saber: unipersonal (administrador único) o pluripersonal (varios administradores, mancomunados o solidarios) ${ }^{15}$.

Tal y como ocurrió con el CUBG analizado anteriormente, las objeciones al sistema de cuotas femeninas no tardaron en surgir. El Consejo de Estado consideró que las entidades a las que se alude en el artículo 75 LOI desarrollan una actividad económica regida por la libertad de empresa (art. $38 \mathrm{CE}$ ), y aunque el desarrollo de dicha sujeta a los titulares de tales entidades -sus accionistas- a un conjunto de requisitos que deben observar para conformar sus órganos de administración, ello no obsta para que, dentro de ese marco, los accionistas constituyan sus órganos de administración en la forma que entiendan más adecuada ${ }^{16}$. Volveremos más adelante sobre esta cuestión.

Por su parte, la Confederación Española de Organizaciones Empresariales (CEOE) señaló que «los únicos parámetros de carácter personal exigibles para acceder a puestos directivos en los consejos de administración deben ser la capacidad y el talento del consejero/a que sea capaz de aportar valor a la empresa desde su propia experiencia» ${ }^{17}$.

Frente a esta afirmación es necesario destacar que la norma del artículo 75 LOI no atenta contra los principios de mérito y capacidad toda vez que «es fina-

${ }_{13}$ El artículo 258 del Real Decreto Legislativo 1/2010, de 2 de julio, por el que se aprueba el texto refundido de la Ley de Sociedades de Capital señala cuáles son las sociedades que pueden formular cuenta de pérdidas y ganancias abreviada, y a sensu contrario, las obligadas a presentarlas de forma no abreviada.

${ }^{14}$ Para un análisis extenso sobre el tema véase: CAmpuzano Laguillo, A., «La presencia equilibrada de hombres y mujeres en los Consejos de administración de las sociedades mercantiles»... op. cit., p. 192 ss; SENENT VIDAL, M. en GARcía Ninet, J. (dir.) Comentarios a la Ley de Igualdad... op. cit., p. 549 ss.; Embrid Irujo, J., «Los aspectos mercantiles de la Ley Orgánica para la igualdad efectiva de mujeres y hombres»... op. cit., p. 326 ss.; MáRQUEZ LOBILlo, P., «Consideraciones acerca de la incorporación de la mujer a los consejos de administración tras la Ley Orgánica de Igualdad»... op. cit., p. 285.

15 Vid. Senent Vidal, M., «El principio de presencia equilibrada en el ámbito del Derecho privado»... op. cit., p. 1297; EMBRID IRUJO, J., «Los aspectos mercantiles de la Ley Orgánica para la igualdad efectiva de mujeres y hombres»... op. cit., p. 335.

16 Dictamen del Consejo de Estado en Pleno, de 22 de junio de 2006.

17 Vid. Iglesias ValCARCE, P.; CEOE-Departamento de Relaciones Laborales: «La nueva Ley de Igualdad. Implicaciones laborales y empresariales. Punto de vista empresarial», Madrid, 2007, p. 18. Citado por SEnEnt Vidal, M. en GARCía Ninet, J. (dir.) Comentarios a la Ley de Igualdad... op. cit., p. 547. 
lidad de esta medida que el criterio prevalente en la incorporación de consejeros sea el talento y rendimiento profesional, ya que, para que el proceso esté presidido por el criterio de imparcialidad, el sexo no debe constituir un obstáculo como factor de elección ${ }^{18}$. De esta forma, se reconoce que corresponde a la propia sociedad apreciar la oportunidad de nombrar como consejero a una mujer o un hombre, primando sus cualidades profesionales antes que su condición sexual ${ }^{19}$.

Si bien es cierto que esta disposición normativa impulsó la adopción de las cuotas femeninas por parte de las empresas españolas, no lo es menos que a un año de que finalice el plazo otorgado a las empresas privadas el porcentaje de mujeres sigue siendo escaso (apenas alcanza el $15 \%{ }^{20}$ ).

Junto con esta situación, el hecho de que la norma no establezca ninguna consecuencia por el incumplimiento de la cuota ${ }^{21}$ hace pensar que estamos muy lejos de cumplir con el objetivo del $40 \%$ de representación femenina en los consejos de administración para el año 2015.

\section{REGULACIÓN NORMATIVA EUROPEA}

\subsection{Antecedentes}

La representación femenina en los consejos de administración y los puestos directivos de las mayores empresas de los Estados miembros de la UE, salvo el caso de Noruega, es realmente preocupante. Según los últimos datos en la materia las mujeres apenas alcanzan el $18 \%$ de participación ${ }^{22}$.

Las razones de esta tradicional invisibilidad de las mujeres en puestos de responsabilidad son múltiples y requieren un enfoque integral para abordar el problema. En este sentido, diversos estudios han concluido que la baja representación femenina en los puestos de decisión económica se debe a diferen-

18 Exposición de Motivos LOI, apartado IV.

19 Vid.: Embrid Irujo, J., «Los aspectos mercantiles de la Ley Orgánica para la igualdad efectiva de mujeres y hombres»... op. cit., p. 344.

${ }^{20}$ Según datos de octubre de 2013 publicados por la Comisión Europea en la página web: http://ec.europa.eu/justice/gender-equality/gender-decision-making/database/business-finance/supervisory-board-board-directors/index_en.htm (consultado el 02.06.2014).

${ }^{21}$ No obstante, algunos autores consideran que de acuerdo con el artículo 10 LOI («los actos y las cláusulas de los negocios jurídicos que constituyan o causen discriminación por razón de sexo se considerarán nulos y sin efecto») podrían entenderse como nulos aquellos nombramientos que se realicen sin tener en cuenta la exigible igualdad constitucional ante la Ley y se base exclusivamente en razones de género. Vid.: SENENT VIDAL, M., «El principio de presencia equilibrada en el ámbito del Derecho privado»... op. cit., p. 1030; MÁRQUEZ LOBILlO, P., «Consideraciones acerca de la incorporación de la mujer a los consejos de administración tras la Ley Orgánica de Igualdad»... op. cit., p. 287.

${ }^{22}$ Vid.: http://ec.europa.eu/justice/gender-equality/gender-decision-making/database/business-finance/supervisory-board-board-directors/index_en.htm, op. cit., (consultado el 14.9.2013). 
tes factores, a saber: a) incertidumbre de los directores sobre la capacidad de las mujeres ejecutivas para desempeñar su trabajo a este nivel debido a su falta de experiencias, b) existencia del mito de que las mujeres con menor frecuencia utilizan estrategias proactivas o no buscan promocionarse en su carrera profesional, c) la permanencia de estereotipos de género que tergiversan los verdaderos talentos de las mujeres líderes y pueden potencialmente minar las contribuciones de dichas a las organizaciones, así como sus propias opciones de ascenso y d) la existencia de procesos de promoción a la alta dirección de las compañías con carácter no formal ${ }^{23}$.

Precisamente, es sobre estas causas que ha venido actuando la UE, por una parte fomentado a la autorregulación y, por otra, adoptando una serie de disposiciones normativas de incentivo para poner remedio a la infrarrepresentación de las mujeres en la toma de decisiones económicas. Así, a partir de los años 80 comienzan a producirse una serie de textos jurídicos en los que, además de reconocerse que en las democracias, el Estado y la sociedad requieren la participación de todos los ciudadanos y ciudadanas, se exhorta a los Estados miembros a que promuevan programas de acción positiva para la integración de la mujer en los sectores político, económico y social ${ }^{24}$.

${ }^{23}$ Vid.: Burke, R., «Women directors: selection, acceptance and benefits of board membership», en Corporate Governance, núm. 3, 1997, pp. 118-125; CARTER, N. Y SILVA, C., «The Myth of the Ideal Worker: Does Doing All the Right Things Really Get Women Ahead?», Catalyst Report, 2011; Catalyst: The Double-Blind Dilemma for Women in Leadership: Damned if You Do, Doomed if You Don't, New York, 2007; OALKLEY, J., «Gender-based Barriers to Senior Management Positions: Understanding the Scarcity of Female CEOs», en Journal of Business Ethics, núm. 27, 2000, pp. 321-334; ALIMOMetCALfe, B., «An investigation of female and male constructs of leadership and empowerment», en Women in Management Review, núm. 2, 1995, pp. 3-8.

${ }^{24}$ Entre estas disposiciones destacan: Resolución A 2-169/88 sobre la mujer y los centros de decisión (DOCE C 262, de 10 de octubre de 1988); I y II Programa de acción sobre igualdad de oportunidades para las mujeres (1982-985 y 1986-1990); III Programa de acción para la igualdad de oportunidades entre mujeres y hombres (1991-1995); Resolución del Consejo, de 27 de marzo de 1995, sobre la participación equilibrada de las mujeres y los hombres en la toma de decisiones (DO C 168, de 4 de julio de 1995); IV Programa de acción comunitaria para la igualdad de oportunidades entre hombres y mujeres (1996-2000); Recomendación 96/694/CE, de 2 de diciembre de 1996, relativa a la participación equilibrada de las mujeres y de los hombres en los procesos de toma de decisión (DO L 319, de 10 de diciembre de 1996); Informe sobre la aplicación de la Recomendación 96/694/CE del Consejo, de 2 de diciembre de 1996, relativa a la participación equilibrada de las mujeres y de los hombres en los procesos de toma de decisión [COM (2000) 120 -no publicado en Diario Oficial-]; Resolución del Parlamento Europeo, de 18 de enero de 2001, sobre el Informe de la Comisión sobre la aplicación de la Recomendación 96/694 del Consejo, de 2 de diciembre de 1996, relativa a la participación equilibrada de las mujeres y los hombres en los procesos de toma de decisión (DO C 262/248, de 18 de septiembre de 2001); V Programa de acción comunitaria para la igualdad de oportunidades entre hombres y mujeres (2001-2005); Plan 
Ciertamente, esta normativa influyó de forma positiva en el aumento de la participación femenina en el sector político, empero, no produjo el mismo efecto en el ámbito económico. Es por ello que, recientemente, se han aprobado una serie de instrumentos, entre los cuales destacan:

a) Estrategia para la igualdad entre hombres y mujeres (2010-2015) ${ }^{25}$

La presente Estrategia constituye el plan de acción de trabajo de la Comisión en materia de igualdad de género para los años 2010-2015. En ella, se consagran acciones con respecto a cinco áreas prioritarias, a saber: independencia económica de las mujeres; salario igual; dignidad, integridad y fin de la violencia sexista; igualdad en la acción exterior e igualdad en la toma de decisiones. En relación a cada área se describen acciones clave para estimular el cambio y obtener progresos.

En el área de la igualdad en la toma de decisiones, la Estrategia reconoce que, a pesar de constituir casi la mitad de la mano de obra y más de la mitad de los nuevos licenciados universitarios de la UE, las mujeres, en la mayoría de los Estados, siguen estando infrarrepresentadas en los procesos y puestos de decisión, en especial en los niveles más altos. Motivo por el cual, se restablece como una acción clave el estudio de iniciativas específicas para mejorar el equilibrio de los sexos en la toma de decisiones, toda vez que «las investigaciones evidencian que la diversidad es rentable, y que hay una correlación directa entre la presencia de mujeres en posiciones directivas y el rendimiento de las empresas».

b) Compromiso europeo: Más mujeres en los Órganos Directivos

A través de este Compromiso, propuesto en marzo de 2011, se busca romper con el denominado «techo de cristal» que sigue impidiendo a las mujeres acceder a los puestos de dirección de las mayores empresas de Europa. A tal efecto, se exhorta a las empresas cotizadas para que se comprometan voluntariamente a aumentar la proporción de mujeres en sus consejos de administración hasta el 40\% para el año 2020.

c) Resolución del Parlamento Europeo sobre las mujeres y la dirección de empresas $^{26}$

En esta Resolución se señala que las medidas proactivas adoptadas por el sector privado con el objetivo de lograr una mayor representación femenina, como las dirigidas a valorizar los recursos humanos en las empresas con mi-

de trabajo para la igualdad entre las mujeres y los hombres (2006-2010); Estrategia para la igualdad entre mujeres y hombres 2010-2015. 2010.

${ }^{25}$ COM (2010) 491 final -No publicada en el Diario Oficial-, de 21 de septiembre de

26 Texto aprobado 2010/2115 (INI). 
ras a realizar un mejor seguimiento de la carrera de las mujeres o la creación de redes que impulsen la participación y la promoción de dichas, han resultado ser insuficientes para transformar su situación en las empresas, ya que siguen estando infrarrepresentadas en los órganos de dirección de éstas. En este sentido, se insta a las empresas a alcanzar el porcentaje crítico de un $30 \%$ de mujeres entre los miembros de los órganos de dirección para 2015 y de un $40 \%$ para 2020 .

d) Resolución del Parlamento Europeo sobre la igualdad entre mujeres y hombres en la $\mathrm{UE}^{27}$

Una vez más, el Parlamento solicita a la Comisión que, ante los pocos avances que se han alcanzado en el año 2011 en cuanto a la implementación de medidas por parte de las Empresas y los Estados miembros tendentes a equilibrar la participación de hombres y mujeres los puestos de responsabilidad de las empresas, se adopten propuestas legislativas concretas que incluyan la introducción de cuotas a fin de aumentar la representación femenina en los consejos de administración hasta el $30 \%$ en 2015 y el $40 \%$ en 2020 , teniendo en cuenta, al mismo tiempo, las responsabilidades de los Estados y sus peculiaridades económicas, jurídicas, estructurales y regionales.

En el ámbito internacional, las Naciones Unidas también ha reconocido que, a pesar de los éxitos alcanzados mediante la integración de principios y acciones a favor de la responsabilidad empresarial, la participación plena de las mujeres en el sector privado -desde el puesto de Director Ejecutivo a la plantilla industrial y la cadena de suministros- sigue siendo una utopía. Por lo cual, considera como necesario que los Estados garanticen una participación femenina suficiente $-30 \%$ o más- en los procesos de toma de decisión y de dirección a todos los niveles y en todos los sectores económicos ${ }^{28}$.

No obstante, estas iniciativas no han aportado ningún avance palpable (apenas se ha logrado en los últimos años una mejora anual de $0,6 \%{ }^{29}$ ). Los datos muestran que existe una gran distancia entre la proporción de mujeres que trabajan y las que están a nivel directivo en todos los Estados. Las mujeres ocupan un cuarto de los escaños en los consejos de las grandes empresas

27 Texto aprobado (2011/2244(INI)), 13 de marzo de 2012.

${ }^{28}$ Principios para el Empoderamiento de las Mujeres. La igualdad es un buen negocio (marzo de 2010).

${ }^{29}$ Empero, es necesario destacar que se produjo una ligera mejora de $1,9 \%$ entre octubre de 2010 y enero de 2012, vinculada al intenso debate público iniciado por la Comisión y por el Parlamento Europeo para acelerar el ritmo de cambio. Vid.: COMISIÓN EUROPEA: Women in economic decision-making in the EU: Progress Report, Luxemburgo, 2012, p. 11. 
que cotizan en Finlandia, Letonia y Suecia, y poco más de una quinta parte en Francia. Hay menos de una de cada 10 en Irlanda, Grecia, Estonia, Italia, Portugal, Luxemburgo, Hungría, menos de una de cada 20 en Chipre y alrededor de una de cada 30 en Malta $^{30}$.

Esta desigual representación femenina en los consejos de administración se debe a que las medidas adoptadas por los Estados no son homogéneas, sino que, por el contrario, se sitúan dentro de un espectro que va desde las cuotas jurídicamente vinculantes acompañadas de sanciones hasta la autorregulación en sectores concretos.

Hasta la fecha, tres Estados miembros (Francia ${ }^{31}$, Italia y Bélgica) han introducido, en 2011, un sistema de cuotas jurídicamente vinculante, que incluye sanciones, siguiendo el ejemplo de Noruega, país pionero en el ámbito de la adopción de cuotas de representación de las mujeres ${ }^{32}$. Países Bajos y España también han adoptado medidas legislativas que prevén cuotas en favor del género menos representado, aunque no se sanciona su incumplimiento. En Dinamarca, Finlandia, Portugal, Grecia, Austria y Eslovenia sólo se aplican cuotas para la representación femenina en los consejos de administración de las empresas de titularidad pública. En los demás Estados, que no han consagrado ninguna medida obligatoria, se han adoptado acciones de carácter voluntario que promueven la representación de las mujeres en los

${ }^{30}$ Ibídem.

${ }^{31}$ En Francia se ha aprobado la Loi 2011-103 relative à la représentation equilibrée des femmes et des hommes au sein des conseils d'administration et de surveillance et à l'égalité professionnelle, de 27 de enero de 2011, por la que se establece una cuota del $20 \%$, a cumplir en el año 2014 y otra del $40 \%$, para el año 2017 . El primero de estos objetivos ya ha sido cumplido y se considera que se logrará el segundo incluso antes del lapso otorgado por la ley. Para un mayor análisis de este tema véase: RoDRÍGUEZ GoNZÁLEZ, A.: Mujeres en los Consejos de administración de las sociedades mercantiles, op. cit.; Rodríguez GonZÁlez, A.: «Ley núm. 2011-103 de 27 de enero, relativa a la representación equilibrada de mujeres y hombres en el seno de los consejos de administración y vigilancia de la igualdad profesional»; en Revista de Derecho de Sociedades, 2011, núm. 36, pp. 539-542.

${ }^{32}$ El caso noruego es el más emblemático a nivel europeo, ya que gracias a la aprobación de una cuota legislativa del $40 \%$ de representación de ambos sexos en los consejos de administración de las empresas, la participación femenina aumentó de forma considerable de $15,9 \%$ en 2004 a $37 \%$ en 2007, y finalmente llegó a la meta del $40 \%$ en 2008 . Actualmente, Noruega es el país europeo con mayor presencia de mujeres en los consejos de las empresas cotizadas. Véase: HoEl, M., «The quota story: five years of change in Norway», en AA. VV. Women on Corporate Boards of Directors: International Research and Practice, Edward Elgar Publishing Limited, Cheltenham, 2008, p. 84 ss.; Comisión Europea: Women in economic decision-making... op. cit., p. 9 ss.; SWEIGART, A., «Women on Board for Chance: The Norway Model of Boardroom Quotas As Tool For Progress in the United States and Canada», en Northwestern Journal of International Law and Business, núm. 4, 2012, pp. 81-115. 
puestos superiores de gestión (por ejemplo: Dinamarca, Alemania, Luxemburgo, Polonia, Suecia y el Reino Unido) ${ }^{33}$.

Estas discrepancias en los ordenamientos jurídicos nacionales han generado, además de un desequilibrio de género en la toma de decisiones económicas según los Estados miembros, un obstáculo al mercado interior al imponer a las empresas cotizadas en la UE unos requisitos divergentes en relación con su gobernanza empresarial. Por lo cual, la Comisión en su Comunicación titulada: «El Equilibrio entre hombres y mujeres en la dirección de las empresas: una contribución a un crecimiento inteligente, sostenible e integrador», considera necesaria la realización de una acción legislativa a nivel de la UE para mejorar el equilibrio de género en los consejos de las empresas cotizadas de forma estructural e irreversible.

En este contexto, se propone la adopción de una Directiva, que tiene por objeto promover la igualdad de género en la toma de decisiones económicas y aprovechar plenamente la totalidad de candidatos capacitados existente, a fin de alcanzar una representación de los sexos más equitativa en los consejos de administración de las empresas, contribuyendo con los objetivos de la Estrategia Europa 2020 34 .

\subsection{Propuesta de Directiva del Parlamento Europeo y del Consejo} destinada a mejorar el equilibrio de género entre los administradores no ejecutivos de las empresas cotizadas y por la que se establecen medidas afines, de 14 de noviembre de $2012^{35}$

Como se ha evidenciado hasta ahora, el interés por alcanzar la igualdad real y efectiva entre hombres y mujeres ha sido, desde hace varios años, un

${ }^{33}$ Vid.: Comisión Europea: Women in economic decision-making in the EU: Progress Report..., op. cit., pp. 17 ss. Además este texto, en los últimos años, la Comisión ha presentado varios estudios en los que se hace un balance de la situación relativa a la diversidad de género en la toma de decisiones económicas en la UE: More women in senior positions. key to economy stability and growth (2010); The Gender Balance in Business Leadership (2011); y, Progress on equality between women and men in 2011 (2012).

34 (COM (2010) 2020 final). La Estrategia reconoce que Europa necesita utilizar plenamente su potencial laboral con el fin de hacer frente a los retos de una población envejecida y al incremento de la competencia mundial. Para ello fija el objetivo principal de alcanzar una tasa de empleo del $75 \%$ de las mujeres y hombres de 20 a 64 años de edad en 2020.

${ }^{35} \operatorname{COM}(2012) 614$ final. Aunque ha generado muchas críticas este documento estratégico ha sido acogido favorablemente por el Comité Económico y Social Europeo a través del Dictamen sobre la Propuesta de Directiva del Parlamento Europeo y del Consejo destinada a mejorar el equilibrio de género entre los administradores no ejecutivos de las empresas cotizadas y por la que se establecen medidas afines, de 9 de mayo de 2012 (2013/C 133/13), y por el Comité de las Regiones en el Proyecto de Dictamen sobre la Directiva destinada a mejorar el equilibrio de género entre los administradores 
objetivo fundamental de la UE. Una muestra de ello es la Propuesta de Directiva destinada a mejorar el equilibrio de género entre los administradores no ejecutivos de las empresas cotizadas y por la que se establecen medidas afines.

Con este texto normativo se pretende avanzar hacia la consecución del equilibrio de género en los consejos de administración de las mayores empresas de los Estados de la UE, por considerar que la no utilización de las competencias de las mujeres altamente capacitadas constituye una pérdida de recursos humanos y, por ende, de potencial crecimiento económico ${ }^{36}$.

A tal efecto, en el artículo 4, se propone la adopción de una medida de discriminación inversa (cuota) cuyo objetivo mínimo es alcanzar una presencia del $40 \%$ del género menos representado entre los administradores no ejecutivos en los consejos de administración de las empresas cotizadas ${ }^{37}$, para el año 2020 (o en 2018 si se trata de una empresa pública).

De esta forma, se obliga a las empresas que se encuentren por debajo de este nivel de representación femenina a realizar nombramientos para estos puestos a través de un procedimiento de selección basado en un análisis comparativo de las cualificaciones de cada candidato, aplicando unos criterios preestablecidos, claros, formulados de forma neutra y carentes de ambigüedades ${ }^{38}$.

no ejecutivos de las empresas cotizadas y por la que se establecen medidas afines, de 30 de mayo de 2013.

36 Esta idea de aprovechamiento de recursos humanos se ha venido reiterando recientemente por parte de las instituciones comunitarias. En este sentido, tanto el Consejo como el Comité Económico y Social Europeo han afirmado que las políticas de igualdad de género son vitales para el crecimiento económico, la prosperidad y la competitividad, por lo cual, es necesario emprender acciones para fomentar la igual participación de las mujeres y los hombres en la toma de decisiones a todos los niveles y en todos los ámbitos, con el fin de aprovechar plenamente todos los talentos. Vid.: Pacto Europeo por la Igualdad entre hombres y mujeres para el 2011-2020, Dictamen del Comité Económico y Social Europeo sobre el tema «La dimensión de género en la Estrategia Europa 2020» 2013/C 76/02.

${ }^{37}$ La Propuesta se centra en las empresas cotizadas, debido a su importancia económica, su mayor visibilidad y a que sientan las normas del sector privado en general. Sin embargo, el Parlamento en su Resolución sobre los efectos de la crisis económica en la igualdad de hombres y mujeres y en los derechos de la mujer (2012/2301 (INI)) insta a la Comisión y a los Estados miembros a que se extienda la aplicación de estas medidas que fomentan el equilibrio entre hombres y mujeres en las pequeñas y medianas empresas.

${ }^{38}$ Dentro de estos criterios, generalmente, suele otorgársele un valor especial a la antigüedad que el candidato tenga en el sector empresarial. Situación que, a nuestro entender, debe ser superada porque afecta en mayor medida a las mujeres que nunca conseguirán cumplir con este requisito hasta tanto no existan políticas de conciliación adecuadas que les permitan desarrollar su carrera profesional en igualdad de condiciones que los hombres. En este sentido, véase: RodríGuez GonZález, A.: Mujeres en los Consejos de administración de las sociedades mercantiles, op. cit., p. 23. 
Para cumplir dichos objetivos se establece que, en primer lugar, las cuotas deben ser vinculantes y, en segundo lugar, deben ir acompañadas de un sistema de sanciones efectivas, proporcionales y disuasorias en caso de incumplimiento, que podrían incluir, entre otras, que un órgano judicial pueda imponer multas administrativas o declarar la nulidad o anulación del nombramiento o la elección de los administradores no ejecutivos que incumplan las disposiciones nacionales (artículo 6).

En la recientemente aprobada Resolución legislativa del Parlamento Europeo sobre la Propuesta de Directiva se amplía este catálogo de sanciones y se incluyen: I) la exclusión de licitaciones públicas; II) la exclusión parcial de la concesión de financiación con cargo a los Fondos Estructurales de la Unión; y, III) la disolución obligatoria de la empresa implicada, dispuesta por un órgano judicial competente, en caso de infracción grave y reiterada ${ }^{39}$.

La regulación de las cuotas se justifica, toda vez que la práctica de los Estados revela que el éxito de este tipo de medidas se ha producido, fundamentalmente, en aquellos países que no han propiciado un modelo de comply or explain, sino que por el contrario, han preferido establecer un catálogo de sanciones en caso de incumplimiento. Así, mientras que en Noruega la participación alcanza el 42\%, en Países Bajos y España, a pesar de haberse adoptado cuotas legislativas, no se ha visto ningún incremento importante en la participación de las mujeres en los puestos de dirección, llegando a contar sólo con un $25 \%$ y $15 \%$, respectivamente ${ }^{40}$.

La Propuesta normativa in comento ha reavivado el debate de la posible inconstitucionalidad de las cuotas femeninas por considerar que atentan contra el derecho de igualdad, de propiedad y de libertad de empresa.

En este punto es necesario dejar claro que, a nuestro juicio, la cuota, en modo alguno, atenta contra el derecho de igualdad ni produce una segunda

${ }^{39}$ Véase: Resolución legislativa del Parlamento Europeo, de 20 de noviembre de 2013, sobre la Propuesta de Directiva del Parlamento Europeo y del Consejo destinada a mejorar el equilibrio de género entre los administradores no ejecutivos de las empresas cotizadas y por la que se establecen medidas afines (enmiendas 39, 60 y 61). A juicio de la Comisión, esta modificación propuesta por el Parlamento es desproporcionada y, por tanto, no debe ser incluida en el texto final de la Directiva. Vid.: Posición de la Comisión sobre las enmiendas del Parlamento Europeo, visto en: http://www.europarl.europa.eu/ oeil/popups/ficheprocedure.do? reference $=2012 / 0299 \% 28 \mathrm{COD} \% 29 \& \mathrm{l}=\mathrm{EN} \#$ tab-0 (02.06.2014)

${ }^{40}$ Vid.: http://ec.europa.eu/justice/gender-equality/gender-decision-making/database/business-finance/supervisory-board-board-directors/index_en.htm (visto el 02.06.2014). En el mismo sentido: Comunicación de la Comisión al Parlamento Europeo, al Consejo, al Comité Económico y Social Europeo y al Comité de las Regiones, titulada "El equilibrio entre hombres y mujeres en la dirección de las empresas: una contribución a un crecimiento inteligente, sostenible e integrador", Bruselas, 2012, $\operatorname{COM}(2012) 615$ final, p. 8 
discriminación injustificada porque no se vulnera el criterio más relevante en la selección de puestos de trabajo, esto es, el mérito. En la Propuesta se consagra expresamente que, habiendo candidatos de ambos géneros con la misma cualificación se dará prioridad al candidato del sexo menos representado a menos que una evaluación objetiva, que tenga en cuenta todos los criterios relativos a la persona, incline la balanza a favor del candidato del otro género ${ }^{41}$.

Asimismo, se exige la introducción de criterios claros, neutros y carentes de ambigüedades en los procedimientos de selección de los cargos de dirección de las empresas, por considerar que, en el ámbito empresarial, a menudo, la escogencia de candidatos se limita a un grupo de personas muy restringido y los administradores no ejecutivos, con frecuencia, se contratan a través de un «círculo de viejos amigos» entre los contactos personales y empresariales de los miembros del consejo. Precisamente, son estas prácticas inadecuadas de contratación para puestos en los consejos las que contribuyen a perpetuar la selección de miembros con características similares.

El sistema de cuotas pone así el peso de la contratación no sobre la mujer en particular, que evidentemente cuenta con todas las capacidades para ocupar un cargo de esta naturaleza ${ }^{42}$, sino en aquellos que controlan el proceso de selección/contratación ${ }^{43}$.

En otro orden de ideas, en lo que al derecho de propiedad y libertad de empresa se refiere, reconoce la Propuesta en su exposición de motivos que, de acuerdo con el principio de proporcionalidad, se justifica la implementación de la cuota toda vez que se centra en los administradores no ejecutivos, los cuales, aun siendo elementos importantes, especialmente por lo

${ }^{41}$ La cuota es así perfectamente compatible con la jurisprudencia del TJUE en materia de acción positiva, que expresa que en algunos casos se puede conceder prioridad al género menos representado en la selección para el acceso al empleo o a la promoción, siempre que el candidato del género menos representado tenga la misma cualificación que su competidor del otro género en términos de adecuación, competencia y rendimiento profesional, que la prioridad no sea automática e incondicional sino que pueda ignorarse si existen motivos específicos que inclinen la balanza en favor del otro candidato individual del otro género, y que la candidatura de cada uno de los aspirantes haya sido objeto de una valoración objetiva que tenga en cuenta todos los criterios relativos al a persona de los candidatos. Asuntos: C-450/93 Kalanke (1995), C-409/95 Marschall (1997), C-158/97 Badeck (2000), C-407/98 Abrahamsson (2000).

${ }^{42}$ Actualmente, las mujeres representan cerca del 60\% de los licenciados universitarios en la UE. Lo que es más relevante, la iniciativa de las facultades de empresariales europeas «Mujeres en el consejo» logró identificar más de 8000 mujeres, altamente cualificadas (cumplen con todos los rigurosos criterios de Gobierno Corporativo según lo definido por las empresas que cotizan en bolsa), con experiencia profesional y dispuestas a asumir una plaza en un consejo de administración. Vid.: http://www.edhec.com/html/ Communication/womenonboard.html\#KLINK (consultado el 8.1.2013).

${ }^{43}$ Vid.: DAhleruP, D., About quotas, 2006. Vid.: http://www.quotaproject.org/aboutQuotas.cfm (visto el 7.1.2013). 
que respecta a la gobernanza de las empresas, no intervienen en la gestión cotidiana de estas.

Sobre este particular, creemos que la disposición es deficiente. Al afectar únicamente a los administradores no ejecutivos la cuota pierde eficacia porque no se aumenta la representación femenina en los verdaderos núcleos de poder de la empresa (donde apenas existe un $12 \%$ de mujeres en toda la UE) ${ }^{44}$.

Originalmente la Comisión recomendó que la cuota abarcase tanto a los administradores ejecutivos como no ejecutivos, estimándose que sería la forma más conveniente para aumentar la participación de la mujer en los consejos, así como para generar beneficios económicos y sociales (evidentemente la incidencia en el derecho de libertad de empresa sería mayor, que no desproporcionada). Sin embargo, y luego de las reticencias que generó esta disposición, la afectación a los administradores ejecutivos se redujo a una mera recomendación.

Resulta cuanto menos curioso, el hecho de que se critiquen las cuotas de género como injerencia a la libertad de empresa, pero se dejen de lado otras cuestiones tanto o más relevantes en este sentido, como son los diferentes instrumentos jurídicos europeos que dan paso a la posibilidad de que los trabajadores tengan derecho no sólo a participar en los consejos de administración, sino a tomar decisiones en las mismas condiciones que el resto de los miembros ${ }^{45}$.

Con todo, es importante resaltar que las cuotas femeninas no obligan a elegir personas determinadas, sino a que quien resulte elegido cumpla con ciertos requisitos que garanticen los diversos intereses y derechos en liza (derecho de información de socios, derecho de las minorías a ser oídas o, incluso, representadas, etc. $)^{46}$.

Finalmente, se incluye en la Propuesta una obligación a los Estados miembros de exigir a las empresas cotizadas que faciliten anualmente información a las autoridades nacionales competentes (dos años después de adoptada la cuota) sobre la representación de géneros en sus consejos. Cuando la

${ }^{44}$ Vid.: http://ec.europa.eu/justice/gender-equality/gender-decision-making/database/business-finance/executives-non-executives/index_en.htm

${ }^{45}$ En este sentido, véase: SENENT VIDAL, M., «Equidad de género en la responsabilidad social empresarial», en Revista del Ministerio de Trabajo y Asuntos Sociales, 2007, núm. 67, pp. 203-211; MERCADER UGUINA, J., «Las nuevas formas de participación de los trabajadores en la gestión de la empresa y la Constitución Europea como telón de fondo», en Revista del Ministerio del Trabajo e Inmigración, 2005, núm. 57, pp. 281-302; EsteBAN VELASCO, G.: «Interés social, Buen gobierno y responsabilidad social corporativa (algunas consideraciones desde una perspectiva jurídico-societaria)», en AA.VV. Responsabilidad social corporativa, Universitat Jaume I, Castellón, 2005, p. 56.

${ }^{46}$ Cfr.: Senent Vidal, M. en García Ninet, J. (dir.) Comentarios a la Ley de Igualdad... op. cit., p. 546. 
empresa no haya alcanzado el objetivo, esta información deberá incluir los motivos por los que no se hayan alcanzado los objetivos o compromisos y una descripción de las medidas que la empresa ha tomado o tiene intención de tomar en el futuro para cumplir con dichos (artículo 5).

\section{COMPARATIVA DE AMBOS TEXTOS NORMATIVOS}

Tanto en el derecho español como en el de la UE se ha regulado, a través de una norma jurídica, la representación equilibrada de mujeres y hombres en los consejos de administración de las empresas. En el caso español, mediante la LOI de 2007 y en el europeo con la Propuesta de Directiva de 2012.

Ambos textos establecen la misma cuota de representación femenina (40\%), sin embargo, el contenido de la Propuesta es mucho más ambicioso puesto que se obliga y no se recomienda como en la LOI, de modo que el incumplimiento de la cuota en el plazo señalado por la disposición acarrearía la posible imposición de multas administrativas, la declaración de nulidad o anulación del nombramiento realizado.

Esta es la principal diferencia entre el caso español y el europeo. La Propuesta, a partir del análisis de la evidencia favorable en otros países, opta por establecer una medida de forzoso cumplimiento obviando así el modelo comply or explain, típico de las reglas de buen gobierno corporativo, que deja a la discrecionalidad de la empresa la observancia o no de la cuota ${ }^{47}$.

También son diferentes las referencias que se realizan al mérito y la cualificación. Mientras que en la Exposición de Motivos de la LOI se hace una

${ }^{47}$ Sin embargo, consideramos que es más provechoso fomentar la presencia equilibrada que sancionar la resistencia a su consecución, y en este aspecto la LOI, prevé una serie de incentivos que no consagra la Propuesta. Así el artículo 34 señala: 1. Anualmente, el Consejo de Ministros, a la vista de la evolución e impacto de las políticas de igualdad en el mercado laboral, determinará los contratos de la Administración General del Estado y de sus organismos públicos que obligatoriamente deberán incluir entre sus condiciones de ejecución medidas tendentes a promover la igualdad efectiva entre mujeres y hombres en el mercado de trabajo, conforme a lo previsto en la legislación de contratos del sector público.

En el Acuerdo a que se refiere el párrafo anterior podrán establecerse, en su caso, las características de las condiciones que deban incluirse en los pliegos atendiendo a la naturaleza de los contratos y al sector de actividad donde se generen las prestaciones.

2. Los órganos de contratación podrán establecer en los pliegos de cláusulas administrativas particulares la preferencia en la adjudicación de los contratos de las proposiciones presentadas por aquellas empresas que, en el momento de acreditar su solvencia técnica o profesional, cumplan con las directrices del apartado anterior, siempre que estas proposiciones igualen en sus términos a las más ventajosas desde el punto de vista de los criterios objetivos que sirvan de base a la adjudicación y respetando, en todo caso, la prelación establecida en el apartado primero de la disposición adicional octava del Texto Refundido de la Ley de Contratos de las Administraciones Públicas, aprobado por Real Decreto Legislativo $2 / 2000$, de 16 de junio. 
breve mención al respecto, en la Propuesta no sólo se hace referencia en el articulado a dichos sino que se consideran como elementos fundamentales a tener en cuenta en el momento de la selección del candidato/a.

Esta disposición cobra importancia, toda vez que, en el ámbito empresarial, las prácticas inadecuadas de contratación para puestos en los consejos de administración han contribuido a perpetuar la selección de miembros con características similares (generalmente del sexo masculino), trayendo como consecuencia el mantenimiento de barreras estructurales que impiden a las mujeres, en comparación con los varones, ocupar altos cargos en las empresas.

Otra discrepancia, que deriva de la propia naturaleza recomendatoria del artículo 75 de la LOI, es el hecho no se establece ningún mecanismo que controle (una vez finalizados los ocho años) el cumplimiento de la cuota en los consejos de administración. Por el contrario, en la Propuesta se incluye una obligación a los Estados miembros de exigir a las empresas cotizadas que faciliten anualmente información a las autoridades nacionales competentes (dos años después de adoptada la cuota) sobre la representación de géneros en sus consejos y las medidas que se hayan tomado para aumentar la presencia femenina en dichos.

En cuarto lugar, en ambos textos se exige mayor cumplimiento de la cuota a las empresas públicas, por considerar que en ellas los Estados ejercen una influencia dominante y, por consiguiente, disponen de más instrumentos para propiciar el cambio con mayor rapidez. Aunado al hecho de que es el Estado el principal garante de la igualdad real y efectiva entre hombres y mujeres.

Por otra parte, a diferencia de lo que ocurre con la LOI que no prevé específicamente los consejeros que se refiere (cuestión que da a entender que puede alcanzar tanto a los dominicales, ejecutivos o independientes) la Propuesta expresa de manera inequívoca que sólo se aplicará a los consejeros no ejecutivos, a fin de limitar la posible afección a la libertad de empresa.

Finalmente, tanto en la LOI (artículo 73) como en la Propuesta (artículo 5) se consagra la posibilidad de que las sociedades adopten voluntariamente medidas adicionales a las cuotas (económicas, asistenciales, comerciales, etc.) destinadas a promover la igualdad de género en el ámbito empresarial.

\section{CONCLUSIONES}

Una vez analizadas las disposiciones sometidas a consideración, es evidente que el modelo de cuotas femeninas en el ámbito empresarial propuesto por la UE es más garantista que el español, toda vez que se trata de una medida obligatoria y no simplemente de una mera recomendación característica de la Responsabilidad Social Corporativa.

En este sentido, en caso de que se apruebe la Propuesta, la normativa española debe abandonar el modelo comply or explain y en su lugar introducir 
una verdadera cuota obligatoria cuyo incumplimiento conduzca a una serie de sanciones. No es que se menosprecie el avance que significó la LOI para la consecución de la igualdad real efectiva entre hombres y mujeres, pero, como hemos señalado, presenta algunas carencias en cuanto al procedimiento previsto por el legislador para lograr la presencia equilibrada en los consejos de administración de las empresas.

En efecto, la experiencia de algunos Estados miembros demuestra que las cuotas obligatorias son necesarias para lograr un avance en la incorporación de la mujer en la toma de decisiones económicas, puesto que, está claro que sin la existencia de dichas las mujeres no llegarían a ciertos puestos de poder, incluso estando mejor cualificadas que sus compañeros varones. El problema no radica en la falta de cualificación femenina, sino en las mentalidades derivadas de una sociedad patriarcal que concibe a la mujer como incapaz de dirigir una empresa por sí misma ${ }^{48}$.

Ciertamente, a través de la adopción de cuotas vinculantes se busca impulsar un cambio en esa mentalidad ${ }^{49}$. Los datos indican que los mayores avances se han producido en aquellos países donde existen medidas legislativas, tales como: Francia que adoptó una cuota vinculante en enero de 2011 y vio la mejora más notable (10\%) de toda la UE y Eslovenia que logró un aumento del $6 \%$ de representación femenina en los consejos de administración. Mientras que en Letonia y Polonia el ascenso no alcanza ni el 2\% ${ }^{50}$.

Junto con estas medidas (tanto a nivel europeo como español) es necesario instaurar un conjunto de acciones específicas dirigidas tanto a la modificación de la cultura organizacional como a desarrollar políticas de conciliación de la vida profesional y la vida familiar ${ }^{51}$. Si únicamente se adoptan las

48 Tal y como expresó la presidenta de la Confederación de empresas de Francia (MEDEF), Laurence Parisot, en un discurso pronunciado ante la Asamblea del Comité Económico y Social Europeo en 2012: «Las cuotas no deberían ser necesarias, pero son la única forma de superar los prejuicios de los hombres hacia las incompetencias de las mujeres». En el mismo sentido véase: Comisión Europea: Report on Progress on equality between women and men in 2012, Bruselas, 2013. COM(2013) 271 final y Comunicación de la Comisión al Parlamento Europeo, al Consejo, al Comité Económico y Social Europeo y al Comité de las Regiones, titulada «El equilibrio entre hombres y mujeres... », op. cit., p. 14.

49 Vid.: Rodríguez GonzÁLez, A.: Mujeres en los Consejos de administración de las sociedades mercantiles..., op. cit., p. 7.

50 Vid.: Comunicación de la Comisión al Parlamento Europeo, al Consejo, al Comité Económico y Social Europeo y al Comité de las Regiones, titulada «El equilibrio entre hombres y mujeres...», op. cit., p. 9-10.

${ }^{51}$ Estas políticas de conciliación son importantes porque mientras que las mujeres sigan condicionadas por su papel de madres, y unido a dicha condición su rol de cuidadoras, difícilmente van a poder ser tomadas en cuenta para incorporarse a los centros de la toma de decisiones (económicas, sociales, políticas, etc.) en igualdad de condiciones que el varón. Ahora bien, dichas medidas deben estructurarse con miras a incluir a la mujer al 
cuotas para promover la contratación o promoción de mujeres en el ámbito empresarial y, al mismo tiempo, no se inician acciones en otros sectores, principalmente la educación y formación profesional, en los que, en la mayoría de los casos, nacen las prácticas y comportamientos discriminatorios, la medida puede convertirse en un remedio insuficiente.

Con la introducción de las cuotas de género en el espacio económico se quiere eliminar de raíz una jerarquía de género «artificialmente creada» y, por lo mismo, «artificialmente modificable». Las cuotas, tal y como se conciben en la Propuesta -respetuosas del principio de mérito y únicamente en casos donde exista una infrarrepresentación del sexo- no atentan contra el derecho de igualdad ni contra la libertad de empresa.

Este tipo de acciones deben ir forzosamente dirigidas a alcanzar un verdadero cambio en las mentalidades, actitudes y conductas que incitan la situación de desigualdad, a fin de situar a todos los miembros de la sociedad (tanto hombres como mujeres) en las mismas condiciones de participación de aquellos bienes que se consideran escasos. En la medida en que se quiebren los antiguos modelos de segregación y jerarquía y se abran oportunidades para todos los grupos y minorías, tendremos una sociedad más justa y equitativa, en la que las cuotas serán un mecanismo totalmente innecesario, pero hasta entonces su adopción, a pesar de suscitar gran controversia, ha demostrado ser precisa y eficaz.

\section{BIBLIOGRAFÍA}

Alimo-Metcalfe, B.: «An investigation of female and male constructs of leadership and empowerment», en Women in Management Review, núm. 2, 1995, pp. 3-8.

BURKE, R.: «Women directors: selection, acceptance and benefits of board membership», en Corporate Governance, núm. 3, 1997, pp. 118-125.

CAmpuzano Laguillo, A.: «La presencia equilibrada de hombres y mujeres en los Consejos de administración de las sociedades mercantiles», en I. García-Perrote Escarín y J. Mercader Uguina (coords.): La Ley de igualdad: consecuencias prácticas en las relaciones laborales y en la empresa, Lex Nova, Valladolid, 2007.

Carter, N. y Silva, C.: «The Myth of the Ideal Worker: Does Doing All the Right Things Really Get Women Ahead?», Catalyst Report, 2011.

Catalyst: The Double-Blind Dilemma for Women in Leadership: Damned if You Do, Doomed if You Don't, New York, 2007.

espacio público pero también al hombre verdaderamente en el ámbito privado, puesto que, en caso contrario, estarían reafirmando el estereotipo de que el cuidado de los hijos corresponde en exclusiva a las madres. SAlazAR Benítez, O.: Cartografias de la igualdad: ciudadanía e identidades en las democracias contemporáneas, Tirant lo Blanch, Valencia, 2010, p. 122. 
Comisión Europea: Women in economic decision-making in the EU: Progress Report, Luxemburgo, 2012.

Comunicación de la Comisión al Parlamento Europeo, al Consejo, al Comité Económico y Social Europeo y al Comité de las Regiones, titulada «El equilibrio entre hombres y mujeres en la dirección de las empresas: una contribución a un crecimiento inteligente, sostenible e integrador», Bruselas, 2012. COM (2012) 615 final.

L Libro Verde de la Comisión de las Comunidades Europeas, «Fomentar un marco europeo para la responsabilidad social de las empresas», Bruselas, 2001, COM (2001) 366 final.

- Report on Progress on equality between women and men in 2012, Bruselas, 2013. COM (2013) 271 final.

DAHLERUP, D.: About quotas, 2006. http://www.quotaproject.org/aboutQuotas.cfm

EMBRID IRUjO, J.: «Los aspectos mercantiles de la Ley Orgánica para la igualdad efectiva de mujeres y hombres», en AA.Vv. Comentarios a la Ley Orgánica 3/2007, de 22 de marzo para la igualdad efectiva de mujeres y hombres, La Ley, Madrid, 2008.

EsteBAn Velasco, G.: «Interés social, Buen gobierno y responsabilidad social corporativa (algunas consideraciones desde una perspectiva jurídico-societaria)», en AA.VV. Responsabilidad social corporativa, Universitat Jaume I, Castellón, 2005.

HoEl, M.: «The quota story: five years of change in Norway», en AA. VV. Women on Corporate Boards of Directors: International Research and Practice, Edward Elgar Publishing Limited, Cheltenham, 2008.

MÁrQuez LobILlo, P.: «Consideraciones acerca de la incorporación de la mujer a los consejos de administración tras la Ley Orgánica de Igualdad», en Revista de Derecho de Sociedades, núm. 32, 2009, pp. 279-291.

Mateu de Ros Cerezo, R.: El Código Unificado de Gobierno Corporativo, Thomson Aranzadi, Navarra, 2007.

MERCADER UGuina, J.: «Las nuevas formas de participación de los trabajadores en la gestión de la empresa y la Constitución Europea como telón de fondo», en Revista del Ministerio del Trabajo e Inmigración, 2005, núm. 57, pp. 281-302.

OALKLEY, J.: «Gender-based Barriers to Senior Management Positions: Understanding the Scarcity of Female CEOs», en Journal of Business Ethics, núm. 27, 2000, pp. 321-334.

RodríGuEZ GonZÁLEZ, A.: «Ley núm. 2011-103 de 27 de enero, relativa a la representación equilibrada de mujeres y hombres en el seno de los consejos de administración y vigilancia de la igualdad profesional»; en Revista de Derecho de Sociedades, 2011, núm. 36, pp. 539-542.

- Mujeres en los Consejos de administración de las sociedades mercantiles: una aproximación al Derecho francés, Valladolid, 2011.

SalazAr Benítez, O.: Cartografías de la igualdad: ciudadanía e identidades en las democracias contemporáneas, Tirant lo Blanch, Valencia, 2010.

SENENT VIDAL, M.: «El principio de presencia equilibrada en el ámbito del Derecho privado», en El levantamiento del velo: las mujeres en el Derecho privado. M. García Rubio y M. Valpuesta Fernández (dirs.), Valencia, 2011.

— «Equidad de género en la responsabilidad social empresarial», en Revista del Ministerio de Trabajo y Asuntos Sociales, 2007, núm. 67, pp. 203-211. 
En J. García Ninet (dir.) Comentarios a la Ley de Igualdad, CISS, Bilbao, 2007. SwEIGART, A.: «Women on Board for Chance: The Norway Model of Boardroom Quotas As Tool For Progress in the United States and Canada», en Northwestern Journal of International Law and Business, núm. 4, 2012, pp. 81-115.

TITLE: Female participation on corporate boards: an approach to the proposal for a Directive of November 2012 on improving male and female balance in companies

RESUMEN: El presente trabajo examina, desde un punto de vista jurídico, la representación femenina en los consejos de administración de las empresas a partir del análisis comparado de la Propuesta de Directiva europea (aprobada, no sin cierta polémica, el pasado 14 de noviembre de 2012) y la normativa española, indicando algunas de sus principales similitudes y diferencias. Asimismo, se estudian los argumentos relativos a la supuesta vulneración del derecho de igualdad y a la libertad de empresa, a fin de estimar la conveniencia de las cuotas para mejorar la situación de la mujer en la toma de decisiones económicas.

PALABRAS CLAVE: cuotas, representación femenina, consejos de administración, igualdad.

ABSTRACT: The present work examines, from the legal point of view, the female representation in the corporate boards in companies. To address this topic a comparative analysis was made between the European Directive Proposal (approved, with some controversy, on November 14 ${ }^{\text {th }}$, 2012) and the Spanish regulation, pointing out some of their similarities and differences. At the same time, arguments about the supposed infringement of the equity and free enterprise rights are studied, in order to estimate the convenience of the quotas for the improvement of the women's situation in the economic decision making.

KEYWORDS: quotas, female representation, corporate boards, equality.

RECIBIDO: 27.09.2013

ACEPTADO: 26.05.2014 